\title{
WSN for AIR Quality Monitoring in Annaba City
}

\author{
Mohamed FEZARI and Mohamed Seghir BOUMAZA1 \\ Laboratory of Automatic and Signals Annaba \\ Badji Mokhtar Annaba University, Faculty of Engineering, BP:12, Annaba, 23000 \\ Annaba, Algeria \\ Ahmed Al-DAHOUD , Ali Al-DAHOUD \\ Al-Zaytoonah University of Amman, Jordan , Faculty of IT, JORDAN \\ Amman, Jordan
}

\begin{abstract}
Wireless sensor networks (WSN) have been involved in different applications including monitoring many environmental phenomena such as air quality assessment, forest fire monitoring, flood rivers control.. In this paper, a WSN architecture where nodes are equipped with gas, temperature and dust sensors and Arduino-uno as microcontroller have been designed for air quality monitoring for some sensible area in Annaba City East of Algeria. The previous design included several units mainly: MSP430 Microcontroller, Gas and dust sensors, and the current regulator circuit. The new design is based on Arduino-Uno as micro-system. Comparing of normal gas levels for the clean air, the obtained results indicate that there is a big difference in the gas levels of both gases (LPG , NO2 and CO) which obtained from the several tests. However, the acquired results for the air quality control in some areas in Annaba city show no risky situation to be considered for further actions. In this work we cover the field of Air quality monitoring electronic Nodes design and wireless transmission of fusion data. Then A GUI has been designed for simulation of the WSN in controlling the environment air Quality. Tests are encouraging; the flexibility off the shelf components and the ease of design facilitates the implementation of this system.
\end{abstract}

Keywords: WSN, AQM, air Quality Index, Environment Monitoring

\section{INTRODUCTION}

Wireless Sensor Networks (WSNs) technology [4] is in the front part of the investigation of the computer networks and it could be the next technologic market of with huge sum of money in investment. Sensor nodes can be fixed or mobile, they have limited processing power, storage, bandwidth, limited wireless transmission range and energy powered by battery. This limitation makes provision of the security in sensor networks not an easy task [4]. The availability of cheap, low power, and miniature embedded processors, radios, sensors, and actuators, often integrated on a single chip, is leading to the use of wireless communications and computing for interacting with the physical world in applications such as air quality control.

Sensor networks may consist of different types of sensors [5] such as seismic, low sampling rate magnetic, thermal, visual, infrared, acoustic and radar, which are able to monitor a wide variety of environment situations [5] such that: temperature, humidity, air quality, vehicular movement, lightning condition, soil makeup, noise levels, the presence or absence of certain kinds of animals or objects, mechanical stress levels on attached motors, and the current characteristics such as speed, direction, and size of an vehicle. A sensor node is made up of four basic components [5] as shown in Figure 2: a of sensing unit, a processing unit, a transceiver unit and a power unit.

In this paper, we propose to use a WSN based microcontroller equipped with gas sensors have been actively used for air quality monitoring. The design included several units mainly: Arduino Microcontroller, MQ-2 Gas Sensors, and the current regulator circuit the paper si organized as follow: in second paragraph after introduction we define primary pollutants, in paragraph 3 , we present the hardware proposition design with main components. In section 4 , format and communication with the special sensor DHT11 is illustrated, then we finish the paper by presenting results ,discussion of simulation and conclusions in section 5 and 6 respectively. 


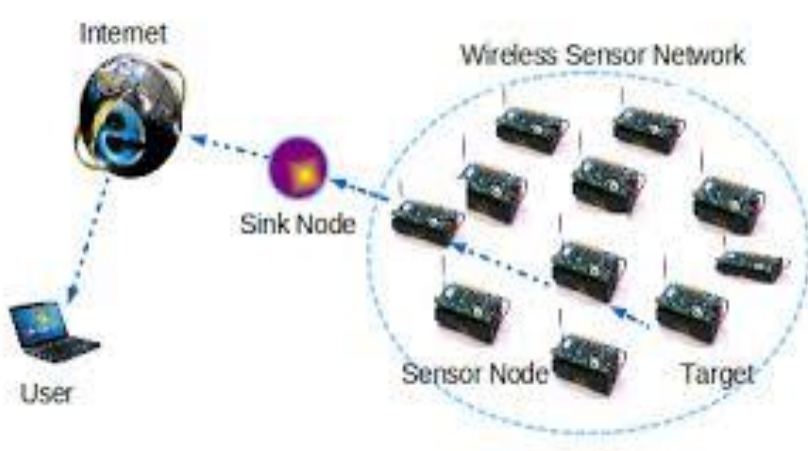

Fig. 1: The Hardware Design Schematic Diagram.

\section{POLLUTANTS}

Primary pollutants are those in which the substance emitted is itself hazardous. Some primary pollutants also produce other dangerous substances after undergoing chemical reactions in the atmosphere, and these are known as secondary pollutants. Primary pollutants include the following substances as mentioned in [15].

Particulates: This includes dust, smoke, aerosols and haze any finely divided airborne solid material. Particulates are commonly generated by fires, motor vehicles, some industries (particularly road building, quarries and fossil fuel power stations) and various natural sources including volcanoes, plant and animal matter and dirt. Particulates are aesthetically displeasing, can irritate the eyes and cause respiratory problems. In recent years concerns have been raised about the possible health effects of 'fine' particulate matter (less than $10 \mu \mathrm{m}$ diameter). These have been shown to be associated with increases in hospitalization and even deaths from respiratory illnesses and heart disease.

Sulphur dioxide, SO2 : Sulphur dioxide is often produced by the industrial processes which produce particulates, the primary sources of SO2 being coal, fuel oil and diesel. Being a corrosive acidic gas, sulphur dioxide damages buildings and other materials, and can cause respiratory problems.

Carbon monoxide, $\mathrm{CO}$ : The commonest source of carbon monoxide is motor vehicle emissions, where it results from the combustion of petrol in the presence of insufficient oxygen. It is also a result of some fuel-consuming industries and domestic fires. Carbon monoxide is a colorless, odorless, highly toxic gas that displaces oxygen in human blood, causing oxygen deprivation.

The oxides of nitrogen, NOx: NOx refers to the mixture of nitric oxide (NO) and nitrogen dioxide (NO2) formed by the oxidation of nitrogen during the combustion of air. The majority of NOx is produced in motor vehicle emissions, although other sources can have significant local impact. NOx is a contributor to several secondary pollutants, and NO2 is a respiratory irritant that can also corrode metals at high concentrations.

Benzene : Over the last few years leaded patrols have been phased out of use. However this has resulted in higher levels of benzene and other aromatics in the substitute unleaded petrol. Benzene breaks down quickly in the environment and is not stored in the tissues of plants or animals. However, it is still hazardous to humans at high levels as it can cause several diseases of the blood including leukemia (cancer of the white blood cells). Benzene monitoring programmes were started in New Zealand in 1994 and are continuing because the levels in some locations were found to be reasonably high.

Hydrogen sulphide, H2S: Hydrogen sulphide is mainly associated with geothermal activity at Rotorua, where it is responsible for the 'rotten eggs' smell, but it is also formed from the anaerobic decomposition of many organic wastes and is a by-product of paper manufacture and leather tanning (see article). It is highly poisonous (more toxic than hydrogen cyanide), and because it initially anaesthetizes the sensory organs it can build up to high concentrations without warning and cause paralysis and then asphyxiation.

Fluorides: These have two main sources: the Comalco aluminum smelter and fertilizer works . Fluorides can have adverse effects on plants, and in some cases concentrate in the leaves so that animals eating the plants ingest significant quantities.

\section{Proposed AIR MONITORING SYSTEM DESIGN}

The complete system design is shown in figure 1, Hardware Design Schematic Diagram. The design is based on nodes and the architecture of the node contains the following major hardware components:

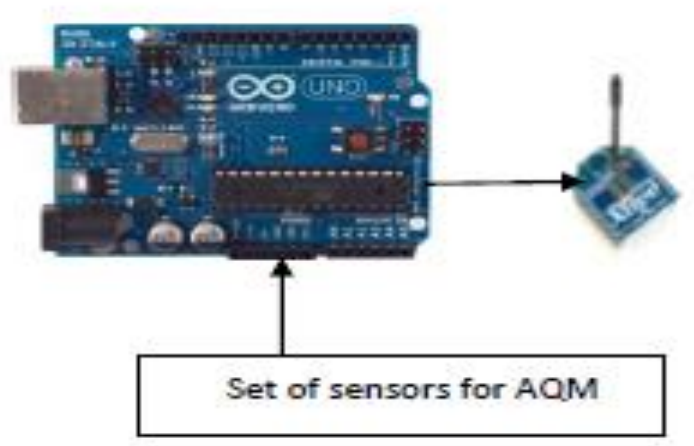

Fig 2.a: Sensor Node main Components 
a. Arduino Microcontroller [1]: this is the core component of the design. Arduino is a flexible programmable hardware platform designed for fast Embedded Systems platform conception. Arduino's little, blue circuit board, mythically taking its name from a local pub in Italy, has in a very short time motivated a new generation of DIYers of all ages to make all manner of wild projects found anywhere from the hallowed grounds of our universities to the scorching desert sands of a particularly infamous yearly arts festival and just about everywhere in between.

Usually these Arduino-based projects require little to no programming skills or knowledge of electronics theory, and more often than not, this handiness is simply picked up along the way. In figure 2.b we can see the main components in the arduino-uno system board.

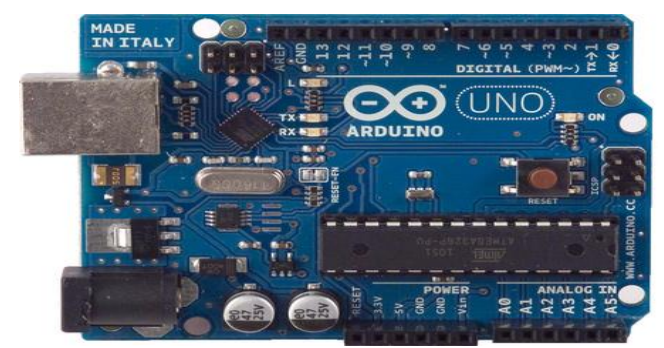

Fig 2.b: Arduino-uno system board

b. MQ-2 GAS Senor [3] Breakout Board: MQ-2 is one of the series of semiconductor Gas Sensors

that is used mainly for gas (such as CO) leak detection for houses, workshops, commercial

building, Fire, Safety detection system as well as a gas leak alarm.

Resistance value of MQ-2 is difference to various kinds and various concentration gases. So, When using this components, sensitivity adjustment is very necessary. we recommend that you calibrate the detector for $1000 \mathrm{ppm}$ liquified petroleum gas $<\mathrm{LPG}>$, or $1000 \mathrm{ppm}$ iso-butane $<\mathrm{i}-\mathrm{C} 4 \mathrm{H} 10>$ concentration in air and use value of Load resistance that ( RL) about 20 $\mathrm{K} \Omega(5 \mathrm{~K} \Omega$ to $47 \mathrm{~K} \Omega)$.

This sensor module utilizes an MQ-2 as the sensitive component and has a protection resistor and an adjustable resistor on board. The MQ-2 gas sensor is sensitive to LPG, ibutane, propane, methane, alcohol, Hydrogen and smoke. It could be used in gas leakage detecting equipments in family and industry. The resistance of the sensitive component changes as the concentration of the target gas changes.

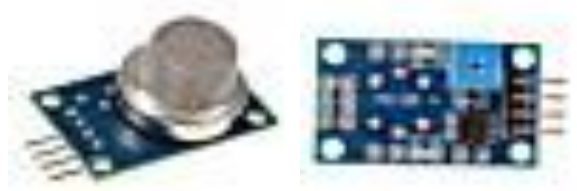

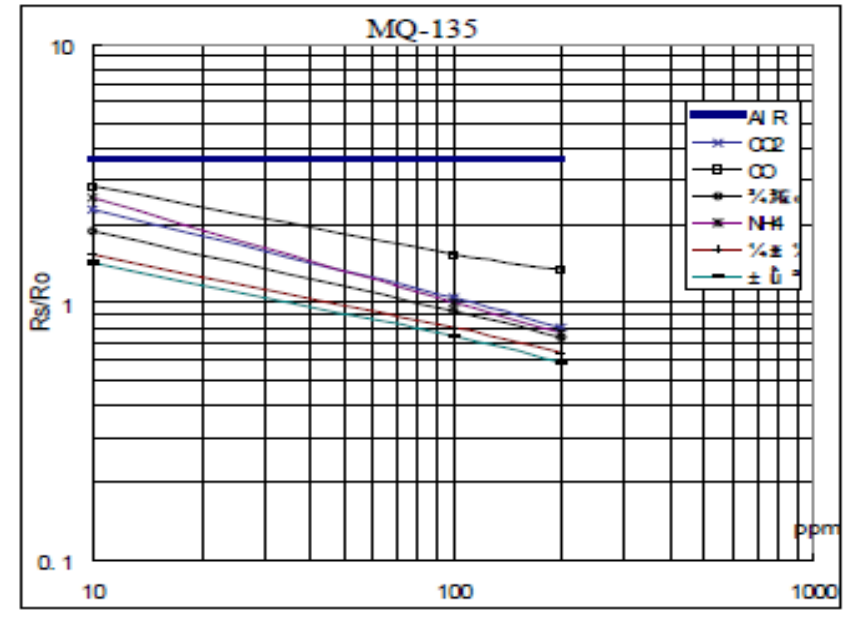

Fig 2.c: Front and rear image of the MQ-135 Gaz sensor; in Blue, potentiometer to control threshold and sensitivity characteristics ofMQ-135 under $\mathrm{T}=22^{\circ} \mathrm{C}$ and $\mathrm{H}=65 \%$, from Technical data MQ-135 sensor.

\section{c Temperature and Humidity sensor}

The DHT11, DHT21 and DHT22 are relative cheap sensors for measuring temperature and humidity. In reference [6] and [7] there is a description of library for reading both values from these sensors. we contacted the manufacturer to get the details of the differences between the two DHT sensors to build a lib that supports both. The DHT21/22 is quite similar to the DHT11 and has a greater accuracy (one decimal) and range (negative temperatures). The hardware pins and handshake are identical but it uses a different data format.

Communication and format for DHT11: Single-bus data format is used for communication and synchronization between MCU and DHT11 sensor. One communication process is about $4 \mathrm{~ms}$.

Data consists of decimal and integral parts. A complete data transmission is 40bit, and the sensor sends higher data bit first.

Data format: 8 bit integral RH data +8 bit decimal RH data + 8bit integral $\mathrm{T}$ data +8 bit decimal $\mathrm{T}$ data +8 bit check sum. If the data transmission is right, the check-sum should be the last 8bit of "8bit integral $\mathrm{RH}$ data +8 bit decimal $\mathrm{RH}$ data +8 bit integral T data + 8bit decimal T data".

d Resistance Circuitry: Resistance value of MQ-2 is difference to various kinds and various

concentration gases. So, When using this components, sensitivity adjustment is very necessary.

we recommend that you calibrate the detector for $1000 \mathrm{ppm}$ liquified petroleum gas $\langle\mathrm{LPG}\rangle$, or

$1000 \mathrm{ppm}$ iso-butane $<\mathrm{i}-\mathrm{C} 4 \mathrm{H} 10>$ concentration in air and use value of Load resistance that ( RL)

about $20 \mathrm{~K} \Omega(5 \mathrm{~K} \Omega$ to $47 \mathrm{~K} \Omega)$. 
e. $A D C$ (analog-to-digital converter): is a device that converts a continuous quantity to a discrete

digital number. Typically, an ADC is an electronic device that converts an input analog voltage (or current) to a digital number proportional to the magnitude of the voltage or current.

The conversion of analog signal and conditioning is performed by Arduino Uno Microcontroller.

f. Light Emitting Diodes: two LEDs used as indicators the Green one indicates the Battery level and the Red one indicates Gas concentration.

g. Trasmission module is based on Zigbee protocol, the Xbee modules are used in transmission and reception, they are also used as gateway node to provide data to the central unit.
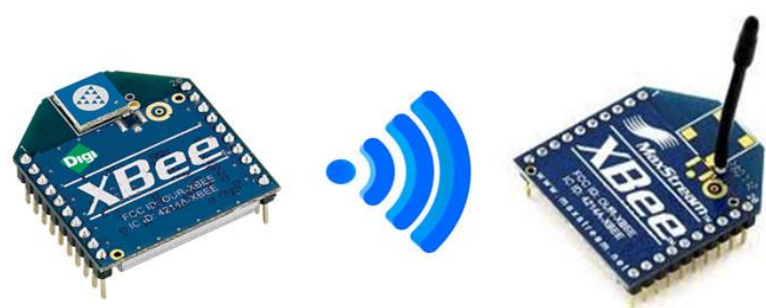

Fig 2.c: Transmission Module Xbee

\section{DHT11 SENSOR PROGRAMMING AND PROTOCOLE}

Single-bus data format is used for communication and synchronization between MCU and DHT11 sensor. One communication process is about $4 \mathrm{~ms}$.

Data consists of decimal and integral parts. A complete data transmission is 40bit, and the sensor sends higher data bit first.

Data format: 8 bit integral RH data +8 bit decimal RH data + 8 bit integral $\mathrm{T}$ data +8 bit decimal $\mathrm{T}$ data +8 bit check sum. If the data transmission is right, the check-sum should be the last 8 bit of "8bit integral $\mathrm{RH}$ data +8 bit decimal $\mathrm{RH}$ data +8 bit integral $\mathrm{T}$ data +8 bit decimal $\mathrm{T}$ data".

Source code for DHT11 sensor reading by Arduino uno: in there code lines we illustrates part of the software to be included into the ardduino uno memory.

\#include "dht.h"

int dht::read11(uint8_t pin)

\{

// READ VALUES

int rv = read(pin, DHTLIB_DHT11_WAKEUP);

if (rv != DHTLIB_OK) humidity = DHTLIB_INVALID_VALUE; // invalid value, or is NaN prefered? value

temperature = DHTLIB_INVALID_VALUE; // invalid

return rv;

\}

\section{// CONVERT AND STORE}

humidity = bits[0]; // bits[1] ==0;

temperature $=$ bits $[2] ; / /$ bits $[3]==0$;

\section{// TEST CHECKSUM}

// bits[1] \&\& bits[3] both 0

uint8_t sum = bits[0] + bits[2];

if (bits[4] != sum) return DHTLIB_ERROR_CHECKSUM;

return DHTLIB_OK;

\}

\section{RESULTS AND DisCUSSION}

The proposed design were used to measure the air quality in several places inside the Annaba City and included different gases levels but focused mainly on measuring three main gases: Carbone Monoxide (CO) and Liquid Petroleum Gas (LPG) and NO2. A sample of obtained results from three different places : clean environment in Seraidi mountains at Annaba , Annaba city center where there is a crowed circulation and El-Hadjar region where a Metal-Steel production firm is installed at $10 \mathrm{Km}$ from Annaba city center, the results are shown in table 1 .

Figure 3 and figure 4, are used to simulate AQM in region in Annaba city by acting on gas concentration levels.

Simulation results: for simulation of WSN nodes, the area is divided into parts where each part can be controlled by a node, in this case the area is divided into 9 regions, and the transmission circuit is chosen so that it can provide the adjacent nodes with the information with minimum consumption of energy.

Scenario 1: by adjusting the sliders for $\mathrm{CO}, \mathrm{SO} 2$ and $\mathrm{NO} 2 \mathrm{Gas}$ we obtained the Red color of the region, which illustrates by node 9 the values sensed: $\mathrm{Co}=206 \mathrm{ppm} \mathrm{SO} 2=160 \mathrm{ppm}$ and $\mathrm{NO} 2=200 \mathrm{ppm}$ 


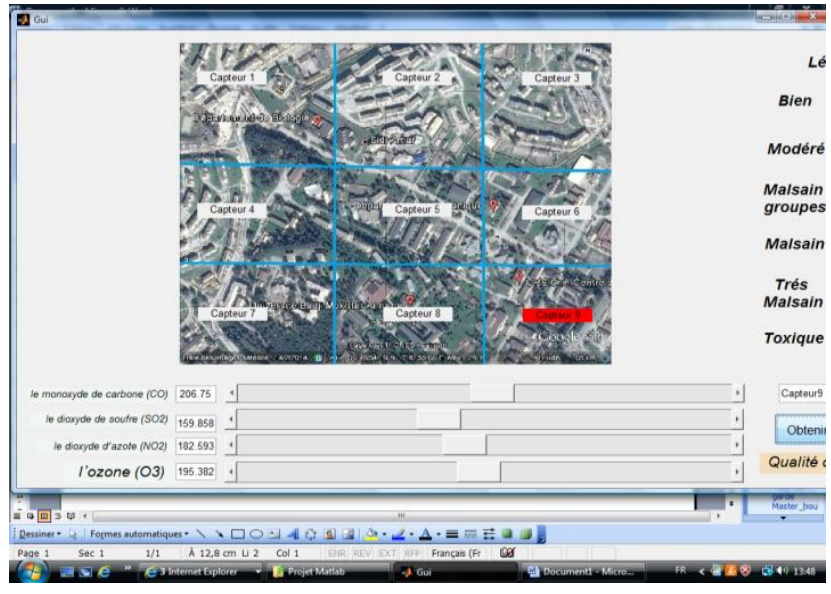

Fig 3: node 9 sensed $\mathrm{Co}=206 \mathrm{ppm} \mathrm{SO2}=160$ ppmand $\mathrm{NO} 2=200$ ppm levels the central control unit

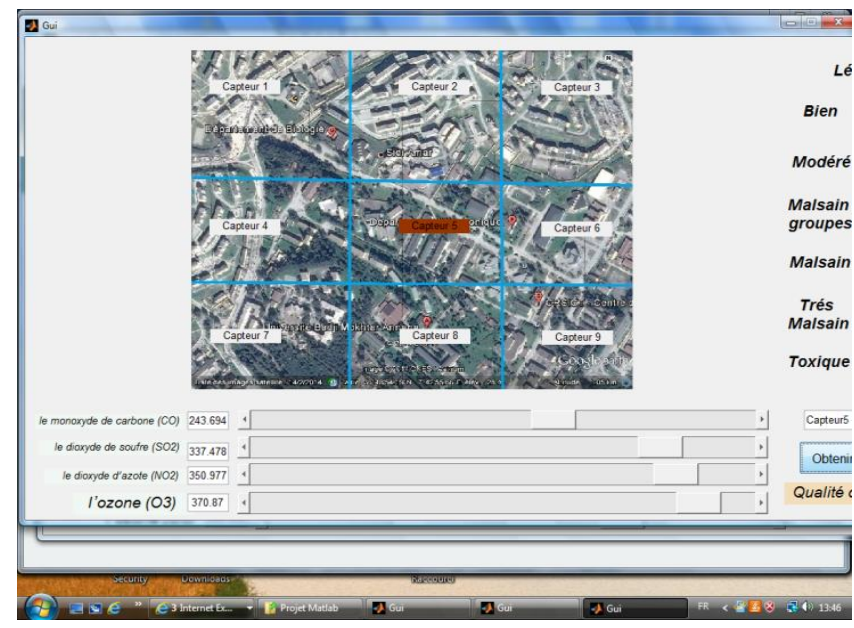

Fig. 4: Concentration of Carbon Monoxide Levels in Clean Air using WSN

Based on the normal gas levels of the clean air [2], the results indicate that there is a big difference in the gas levels of both gases (LPG and CO) which obtained from the several tests and circuit runs. However, the acquired results show no risky situation to be considered for further actions.

\section{CONCLUSIONS}

AIR Quality monitoring System Design to asses the pollution of air in some parts of Annaba city using a microsystem, as a node in Wireless Sensor Network (WSN), is proposed in this article. WSN enhanced the process of monitoring many environmental phenomena such as the air pollution monitoring issue in proposed this paper. It provides a real-time information about the level of air pollution in different regions, as well as provides alerts in cases of drastic change in quality of air. Based on collected information, such data can then be used by the authorities to take prompt actions such as evacuating people or sending emergency response team. The proposed design is enhanced by several ways such as: selecting adequacies' sensors, calibrating these sensors for gas detection, integrating them in a WSN system controlled by an Arduino-Uno, and finally transmission to the central unit using Xbee modules. A Graphic used interface has been presented in this work to simulate the effect of sensors on selected area. The results are interesting, improvements can be done: in providing a web service page that can provide these data to users, as well as more sophisticated sensors could be used such as MQ-135, MQ-136 and others.

\section{Acknowledgements}

Authors appreciate the support of LASA laboratory at Badji Mokhtar Annaba University (BMAU), faculty members of Environment department (BMAU) and Dean of Faculty of IT at Al-Zaytoonah University AMMAN, JORDAN.

\section{REFERENCES}

[1] Mobile Air Quality Monitoring Network at www.isis.vanderbilt.edu/projects/maqumon.

[2] S. Choi, N. Kim, H. Cha, and R. Ha " Micro Sensor Node for Air Pollutant Monitoring: Hardware and Software Issues ". Sensors 2009.

[3]. Technical Data For MQ-2 Gas Sensor, Website http://www.seeedstudio.com/depot/datasheet/MQ-2.pdf

[4] Qasem Abu Al-Haija, "Toward Secure Non-Deterministic Distributed Wireless Sensor Network Using Probabilistic Key Management Approaches", Journal of Information Assurance and Security 6 (2011) 010-018.

[5] I.F. Akyildiz, W. Su, Y. Sankarasubramaniam, E. Cayirci, " Wireless sensor networks: a survey", Computer Networks 38 (2002) 393-422, elsevier.

[6] http://playground.arduino.cc/Main/DHT11Lib

[7] hhtp://www.micro4you.com/files/sensor/DHT11.pdf

[8] Raja Vara Prasad Y. et al,"Real Time Wireless Air Pollution Monitoring System”,ICTACT Journal on Communication Technology,June 2011.

[9] M. Riley, " Programming Your Home: Automate with Arduino, Android, and Your Computer", The Pragmatic Programmers, 2012.

[10] R.A.Roseline, Dr.P.Sumathi, "Local Clustering and Threshold Sensitive routing algorithm for Wireless Sensor Networks", in the IEEE sponsored International Conference on Devices Circuits and Systems(ICDCS'12),March 2012.(Available online at ieeexplore.com).

[11]. Honicky, R.; Brewer, E.A.; Paulos, E.; White, R. N-smarts: networked suite of mobile atmospheric real-time sensors. In Proceedings of the 2 nd ACM SIGCOMM Workshop on Networked Systems for Developing Regions, Seattle, WA, USA; ACM: Seattle, WA, USA, 2008.

[12]. Volgyesi, P.; Nadas, A.; Koutsoukos, X.; Ledeczi, A. Air Quality Monitoring with SensorMap. In Proceedings of the 7th International Conference on Information Processing in Sensor Networks, St. Louis, MO, USA; IEEE Computer Society: St. Louis, MO, USA, 2008.

[13]. So, S.; Koushanfar, F.; Kosterev, A.; Tittel, F. LaserSPECks: laser SPECtroscopic trace-gas sensor networks - sensor integration and applications. In Proceedings of the 6th International Conference on Information Processing in Sensor Networks, Cambridge, MA, USA; IEEE Computer Society: Cambridge, MA, USA, 2007.

[14] Sharma, A.; Golubchik, L.; Govindan, R. On the Prevalence of Sensor Faults in Real-World Deployments. In 4th Annual IEEE Communications Society Conference on Sensor, Mesh and Ad Hoc Communications and Networks, San Diego, CA, USA; IEEE Computer Society: San Diego, CA, USA, 2007. 
ICIT 2015 The 7th International Conference on Information Technology

doi:10.15849/icit.2015.0042 C ICIT 2015 (http://icit.zuj.edu.jo/ICIT15)

[15] Saitas, Jeff; Ground-Level Ozone; [Online] Available http://www.tnrcc.state.tx.us/air/monops/ozoneinfo.html; February 20, 1997.
[16] Xintaras, Charlie and Perry, Mike; Agency for Toxic Substances and Disease Registry; [Online]

http://atsdrl.atsdr.cdc.gov:8080/toxfaq.html; February 20, 1997

TABLE 1: SITUATION OF AIR POLLUTION IN THREE DIFFERENT AREAS IN ANNABA CITY

\begin{tabular}{|c|c|c|c|c|c|c|c|c|}
\hline $\begin{array}{c}\text { Clean Air } \\
\text { Co }\end{array}$ & $\begin{array}{c}\text { Clean Air } \\
\text { NO2 }\end{array}$ & $\begin{array}{c}\text { Clean Air } \\
\text { LPG }\end{array}$ & $\begin{array}{c}\text { Center } \\
\text { Co }\end{array}$ & $\begin{array}{c}\text { Center } \\
\text { NO2 }\end{array}$ & $\begin{array}{c}\text { Center } \\
\text { LPG }\end{array}$ & $\begin{array}{c}\text { Metal- } \\
\text { Steel } \\
\text { Co }\end{array}$ & $\begin{array}{c}\text { Metal- } \\
\text { Steel } \\
\text { NO2 }\end{array}$ & $\begin{array}{c}\text { Metal- } \\
\text { Steel } \\
\text { LPG }\end{array}$ \\
\hline 0.05 & 1 & 2.05 & 20 & 16 & 75 & 35 & 5 & 25 \\
\hline 0.8 & 2.5 & 3.5 & 26 & 13 & 86.9 & 34 & 57 & 24 \\
\hline 0.75 & 0.8 & 2.7 & 24 & 17 & 87.4 & 36.7 & 58 & 38 \\
\hline 0.48 & 0.8 & 1.9 & 26.78 & 15.68 & 80.6 & 40.58 & 55.8 & 26.25 \\
\hline 0.87 & 2.4 & 2.9 & 27.58 & 19 & 76 & 32.78 & 50.15 & 27.8 \\
\hline 0.79 & 1.7 & 3.04 & 29.15 & 20 & 79 & 31.99 & 52 & 30 \\
\hline 0.61 & 1.5 & 2.9 & 30.15 & & 78.95 & 32.58 & 53 & 30.5 \\
\hline
\end{tabular}

